\title{
ENHANCING THE COVERAGE OF INDOOR RADIO LOCALIZATION BY DISTRIBUTED COMPUTATIONS
}

\author{
Andreas Fink, Helmut Beikirch \\ Department of Computer Science and Electrical Engineering, Rostock University \\ A.-Einstein-Str. 2, D-18059 Rostock, Germany \\ \{andreas.fink | helmut.beikirch\}@uni-rostock.de \\ www.igs.uni-rostock.de
}

\begin{abstract}
The prevalent evaluation criterion for indoor local positioning systems (ILPS) is the achievable accuracy in terms of Euclidean distance between estimated and true position. Systems relying on received signal strength (RSS) ranging often use a distributed collection of RSS sensor data at reference nodes and a centralized position estimation. For this direct remote positioning, the accuracy is dependent on the reference node density and thus, is indirect proportional to the achievable coverage. To split up the dependency between these two criteria, we propose a distributed weighted centroid localization (dWCL) strategy with a hierarchical sensor data field bus. Accuracy and coverage of centralized and distributed WCL algorithms are compared for a one-dimensional tracking simulation and 196 reference nodes, arranged in up to 28 gateway segments. Using distributed computations, the localization system's coverage is increased by factor ten while the location estimation error increases only slightly. Copyright (C) Research Institute for Intelligent Computer Systems, 2013. All rights reserved.
\end{abstract}

Keywords: Centroid Localization, Distributed Computing, Human Tracking, RSS Ranging.

\section{INTRODUCTION}

The establishment of an active protection system for the monitoring of personnel in underground coal mines is crucial to save human lives and therefore, is an essential part of the development strategy of leading mining companies. The objective for an indoor local positioning system (ILPS) is to cover the whole area with the same quality of service, determined by the localization accuracy.

In underground coal mines, three different mining methods are used which define the structure of the mining area [1]:

- Cut-and-fill mining is used for irregular coal zones with steep dips. The sectional mining area consists of several types of tunnels.

- Room and pillar mining is used for relatively flat-lying coal deposits, e.g. following a particular stratum. The mining area looks like a large room with randomly shaped pillars.

- Longwall mining is used if the coal seam has a large and thin shape. This specialized type of mining process is characterized by minimal manual handling and highly productive automated control systems.

For longwall mining, the complexity of the selfadvancing mining equipment, e.g. the electro- hydraulic shields for ceiling, is a mortal danger for maintenance staff. A direct remote positioning [2] for the longwall application using the received signal strength (RSS) of radio signals is shown in Fig. 1.

A set of fixed reference nodes receive the RF packets of mobile blind nodes and transmit the corresponding RSS values to a central data concentrator [3]. For the data backbone, the wellknown and failsafe CAN bus is used which offers line-topologies up to the kilometers range and data rates up to $1 \mathrm{Mbit} / \mathrm{s}$ [4]. At the data concentrator, the user positions are calculated and used to intervene in the control system of the mining process and stop regarding shields.

The number of shields which have to be blocked depends on the accuracy and availability of the localization system. RF diversity for an improved availability of radio localization has been analyzed and successfully implemented in [5]. The localization accuracy is dependent on the reference node density and thus, is indirect proportional to the achievable coverage. Since a certain accuracy is required for a productive mining, the maximum distance between the reference nodes is limited, e.g. to a few meters for sub-meter accuracy [3]. For the used CAN bus, the maximum number of nodes on a bus segment is limited to 32 due to bus load 
constraints of typical transceiver circuits [4]. which is not sufficient for the $300 \mathrm{~m}$ longwall Assuming a reference node distance of $3 \mathrm{~m}$, this means an overall system coverage of only $96 \mathrm{~m}$

application, shown in Fig. 1.

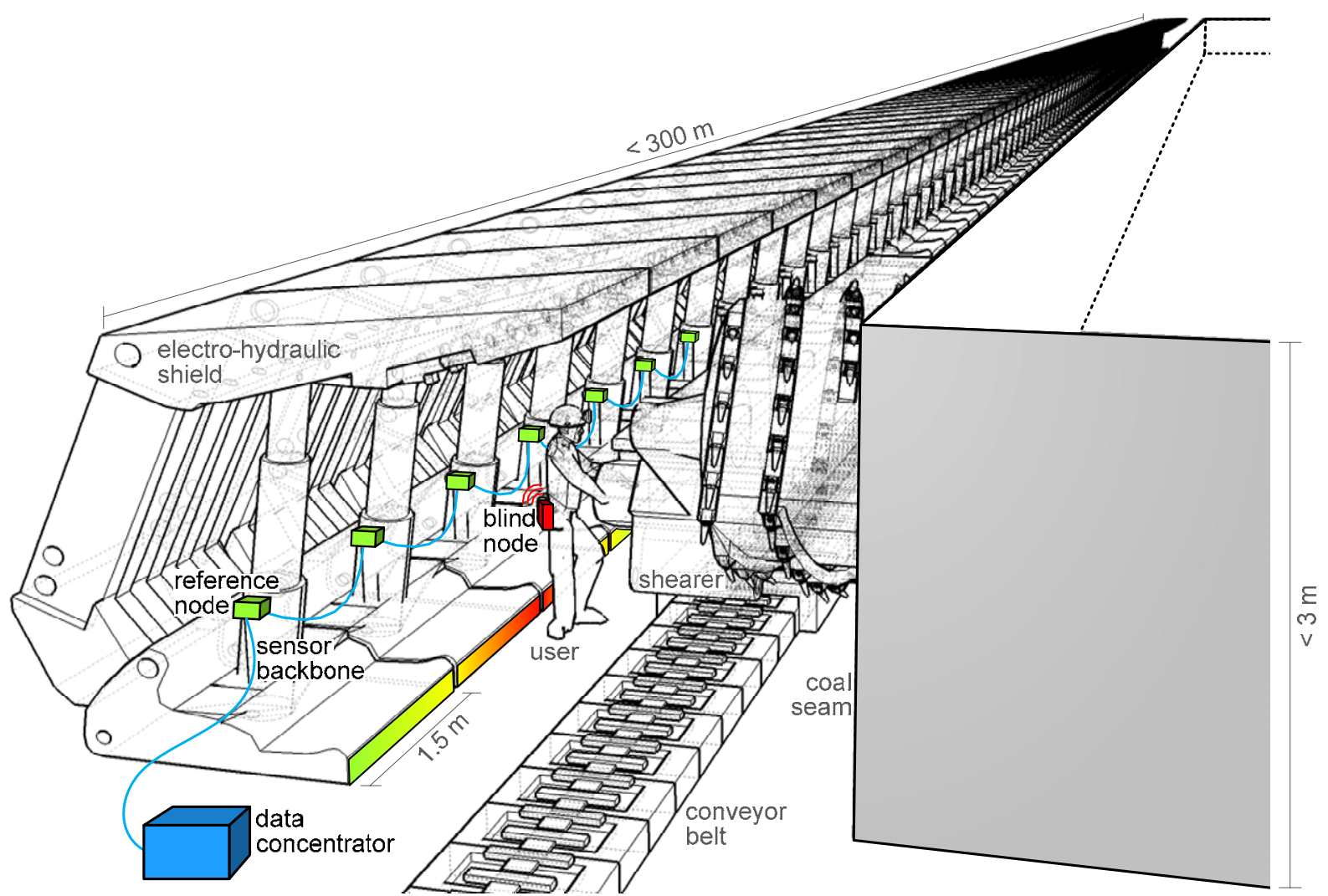

Fig. 1 - Indoor radio localization for personnel tracking in underground longwall coal mining using direct remote positioning ( 20 users along $300 \mathrm{~m}$ ).

To break up the dependency between the two criteria accuracy and coverage, we propose a distributed weighted centroid localization (dWCL) strategy with a hierarchical sensor data field bus in [6]. In the current paper, we enhance the underlying RSS simulation model and investigate the accuracy limits of RSS-based localization.

The remainder of the paper is organized as follows. In section 2, the general classification of indoor localization and a taxonomy of RSS-based positioning are presented. The radio propagation model and consequential accuracy issues are analyzed in section 3, in particular for the propagation in tunnels. In section 4 , the localization system's coverage is deduced from the CAN specifications, especially addressing practical CAN cable lengths and data rates for centralized and distributed location estimation strategies. The distributed weighted centroid localization (dWCL) approach is presented in section 5 . In section 6 , we compare the centralized and distributed localization performance using a one-dimensional tracking simulation. In the last section 7 , the results are discussed and investigated in terms of an outlook for further system developments.

\section{INDOOR RSS LOCALIZATION}

Modern indoor local positioning systems for the tracking and navigation of people and objects show a variety of applied sensor technologies [7]. The main evaluation criteria are the accessible coverage and accuracy of the position estimations. An overview of available technologies with a taxonomy according to these two criteria is deduced from [8] and shown in Fig. 2. Of course, also other criteria like costs for installation and maintenance, the scalability, the user acceptance and specific system limitations should be taken into account. A further taxonomy of localization techniques and system implementations according to these criteria are proposed in [9] and [10].

In Fig. 2, the desired coverage and accuracy for the personnel tracking in the longwall mining is highlighted. Furthermore, the dashed graphs indicate systems with manual sampling like geodetic surveying systems. The dotted graphs indicate systems for typical outdoor use, while their use in indoor scenarios is limited due to intensive signal attenuations. The light solid graphs indicate systems which are limited to line-of-sight (LOS) scenarios. 
All shaded graphs indicate systems which are more or less applicable for non-line-of-sight (NLOS) conditions.

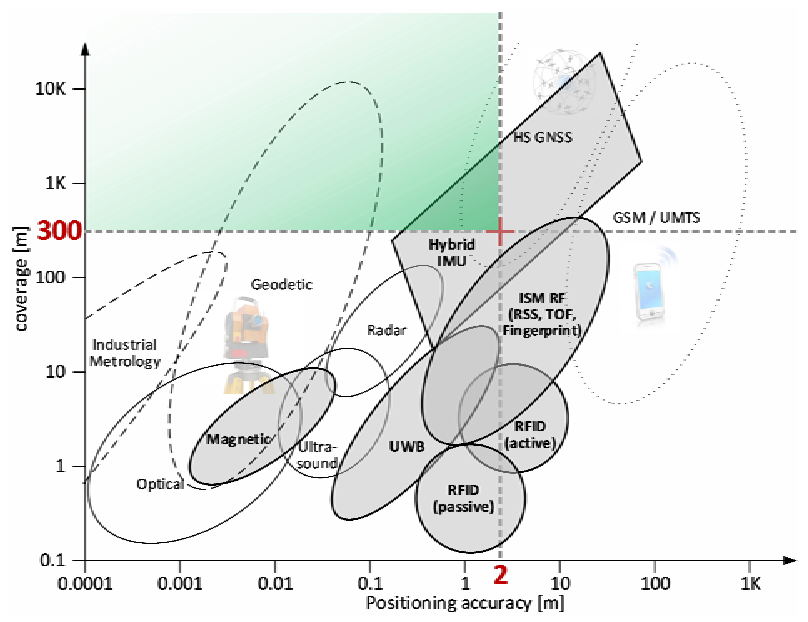

Fig. 2 - Overview of common localization sensor techniques with a classification according to coverage and accuracy, cf. [8].

For harsh indoor environments like a longwall coal mine, the received signal strength (RSS) of RF waves, e.g. using the worldwide harmonized 2.4 $\mathrm{GHz}$ ISM band, is a low-cost but valuable rangebased localization sensor [11]. The performance of RSS-based localization is given by the spider chart in Fig. 3. As also stated in Fig. 2, accuracy and coverage are at medium level. The low-cost hardware and low algorithmic complexity together with low communication overhead enable an overall low cost solution.

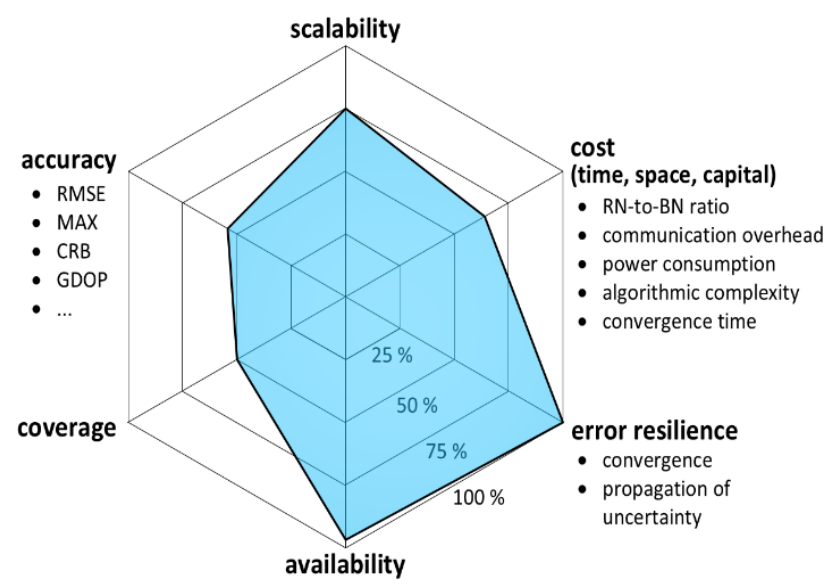

Fig. 3 - Taxonomy of RSS positioning.

\section{RSS MODEL AND ACCURACY LIMITS}

To find a relationship for the distance-dependent RSS and to compare the accuracy of centralized and distributed position estimation strategies in multipath fading environments, it is necessary to have a look at the indoor radio signal propagation.
In this context, the limitations of RSS-based localization will be discussed using latest research results from literature and own path loss experiments.

\subsection{LARGE-SCALE PATH LOSS}

For both, indoor and outdoor radio channels, the distance-depending average path loss shows a logarithmic dropping of power with a linear increasing distance according to the LOS graph, shown in Fig. 4. The log-distance path loss model [12] is used to describe the average power at the receiver. With (1) the average path loss $P L(d)$ (in $\mathrm{dB}$ ) over a LOS distance $d$ between transmitter (TX) and receiver (RX) is given by the reference path loss $P L\left(d_{0}\right)$ over a reference distance $d_{0}$ and the environment-specific propagation coefficient $n$.

$$
P L(d)=P L\left(d_{0}\right)+10 n \log \left(\frac{d}{d_{0}}\right)
$$

The value of $P L\left(d_{0}\right)$ is influenced by the effective radiated power (ERP) of the RF transmitter and the gain of the transmitting and receiving antenna [6]. The slope of the path loss is given by $n$ and is influenced by the specific environmental propagation conditions and the used frequency as given by experiment results in Table 1.

Table 1.Path loss coefficients for typical indoor environments.

\begin{tabular}{|c|c|c|}
\hline Frequency & Reference & PL coeff. $n$ \\
\hline $900 \ldots 1900 \mathrm{MHz}$ & Office [12] & $2.4 \ldots 2.6$ \\
\hline $2000 \mathrm{MHz}$ & Office [13] & $2.5 \ldots 4.0$ \\
\hline $2400 \mathrm{MHz}$ & Office [14] & $1.9 \ldots 3.8$ \\
\hline $5500 \mathrm{MHz}$ & Office [14] & $1.7 \ldots 5.0$ \\
\hline $868 \mathrm{MHz}$ & Factory hall [5] & $2.1 \ldots 2.9$ \\
\hline $1300 \mathrm{MHz}$ & Factory hall [15] & $1.9 \ldots 2.4$ \\
\hline $2440 \mathrm{MHz}$ & Factory hall [5] & $1.9 \ldots 3.0$ \\
\hline $868 \mathrm{MHz}$ & Corridor & $2.4 \ldots 2.8$ \\
\hline $2440 \mathrm{MHz}$ & Corridor & $1.8 \ldots 2.3$ \\
\hline
\end{tabular}

In [15] and [12], values for $n$ between 1.9 and 2.6 are given for frequencies between $900 \mathrm{MHz}$ and $1900 \mathrm{MHz}$ in obstructed indoor environments where not only NLOS conditions but also multipath signal fading affects the RF signal propagation. For 2000 $\mathrm{MHz}$ radio signals in an obstructed office environment, values between 2.5 and 4.0 are given in [13]. In the experimental investigations in [5], the path loss coefficient was determined with $n=2.31$ for $868 \mathrm{MHz}$ and $n=2.62$ for $2400 \mathrm{MHz}$. All these values for the propagation coefficient were found by experiment and are typical values for spacious 
indoor fading environments. They all show the general behavior of an increasing path loss and larger uncertainties for larger frequencies.

The mayor implication of the average path loss for the RSS-based ranging is an imbalanced resolution of the RSS over the distance. With usual receiver hardware, the RSS is sampled over a certain part of the received packet and stored as an 8 bit RSS indicator (RSSI) value which directly scales with the signal strength with $1 \mathrm{dBm}$ resolution [16].

For RF communication systems with the purpose of data transmission like WLAN, the slope should be very flat to guarantee the same quality of operation over a large area. For RSS-based ranging and localization the opposite behavior is preferable [17].

Depending on the distance to the transmitter, two neighboring RSSI will translate into completely different distances. Near the transmitter, where the slope of the path loss graph is very steep, the $1 \mathrm{dBm}$ resolution corresponds to a small distance in the centimeters range. Thus, the limited resolution will not lead to a downgrading of localization accuracy. Looking at the $2440 \mathrm{MHz}$ path loss in Fig. 4, the steep slope from $1 \mathrm{~m}$ to $5 \mathrm{~m}$ is well suitable for RSS ranging. Far away from the transmitter, the slope is very flat and $1 \mathrm{dBm}$ deviation corresponds to several meters RX-TX separation. The $2440 \mathrm{MHz}$ path loss in Fig. 4 shows this flat behavior without larger signal dropouts for distances between $16 \mathrm{~m}$ and 24 $\mathrm{m}$. Here, the resolution of the range measurements will lead to a coarse grained location estimation.

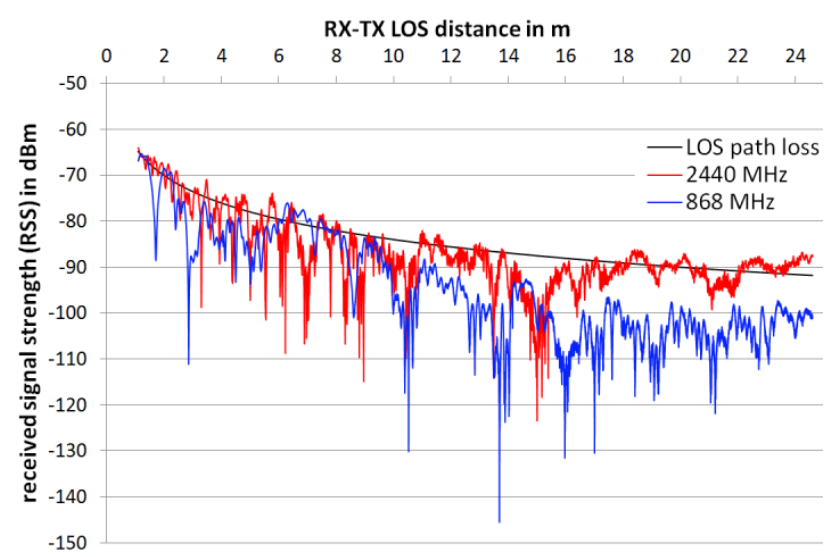

Fig. 4 - Path loss for $868 \mathrm{MHz}$ and $2440 \mathrm{MHz}$ in a narrow corridor.

\subsection{SMALL-SCALE FADING}

The distance dependent path loss from (1) is an average value and therefore not suitable to entirely describe a real channel. In general, a Rayleigh distribution of the RSS is assumed for NLOS conditions. When there is also a dominating LOS component, the fading is modeled with a Rician distribution. The probability density function of the Rician distribution is given by

$$
f(x, v, \theta)=\frac{x}{\theta^{2}} \exp \left(\frac{-\left(x^{2}+v^{2}\right)}{2 \theta^{2}}\right) I_{0}\left(\frac{x v}{\theta^{2}}\right) \text {, }
$$

where $\theta$ is the amplitude of the superimposed RSS from all multipath components and has a scaling functionality. The parameter $v$ is the peak amplitude of the LOS component. For $v=0$, the Rician distribution reduces to a Rayleigh distribution. Thus, the Rician distribution is a generalization of the Rayleigh distribution. For $v \rightarrow \infty$, the distribution reduces to a Gaussian distribution. The ratio of the LOS component compared to the superimposed signal can be expressed by the Rician K-factor according to

$$
K(d B)=10 \log \frac{v^{2}}{2 \theta^{2}} d B,
$$

The environment-specific values of $v$ and $\theta$ can be evaluated with an empirical analysis of multiple path loss observations using a two moment-based estimator as described in [18].

Several implications for RSS-based ranging and localization arise from the small-scale signal behavior. It is obvious, that the signal distribution in random environments also is random and the relation between distance and RSS could not be expressed by an equation [19]. Even with a training of the path loss coefficient $n$, RSS localization is known to be error-prone in multipath environments. The expected performance bound for a wide variety of algorithms shows a median location estimation error of three to four meters [20]. For changing environments, an automatically adjustment of the path loss coefficient should be used [21].

For RSS, the computational complexity of the location estimation is not proportional to the gained accuracy. Especially fine grained algorithms like least squares or maximum likelihood approaches show poorer accuracies than approximative ones [22]. Weighted centroid localization, for example, only needs a few computations and is especially suitable for erroneous range-sensors [3].

\subsection{PROPAGATION IN TUNNELS}

For the application of personnel tracking in longwall mining the environment can be seen as a tunnel with a uniform circular cross-section. When the diameter of the tunnel is small, it can be regarded as an over-sized imperfect waveguide. The propagation will exhibit the guided wave 
characteristics and the propagation loss can be even smaller than that in free space [23].

Some additional assumptions for the small-scale behavior have to be considered. As for other indoor environments with a dominating LOS component, the fast fluctuations are modeled with a Rician distribution [24]. In [1], experiments with $450 \mathrm{MHz}$ and $900 \mathrm{MHz}$ support the theoretical waveguide model. The fast fluctuations are only apparent in the field near the transmitting antenna. As the frequency increases, the fast fluctuating region is prolonged.

Since the waveguide effect is larger for smaller wavelengths, the general large scale behavior of an increasing path loss for larger frequencies does not hold in tunnel environments. The waveguide acts as a high pass filter, so signals with higher frequency attenuate slower. This effect is examined in [1] and also by our experiment results in a narrow corridor. In Fig. 4, we compare the resulting path loss for $2440 \mathrm{MHz}$ and $868 \mathrm{MHz}$. For comparison, both graphs are matched to the same reference path loss $P L(1 \mathrm{~m})=-67 \mathrm{dBm}$. For $2440 \mathrm{MHz}$, the path loss shows a slighter dropping than for $868 \mathrm{MHz}$. For distances above $20 \mathrm{~m}$, the path loss is even lower than the LOS path loss for free-space propagation.

To sum up the influence of indoor radio propagation on the accuracy of RSS-based ranging and localization, in particular for one-dimensional environments like mining tunnels, we propose the following guidelines:

- For tunnels with waveguide characteristics, the larger frequency has a better communication range which is non-intuitive when looking at outdoor and open space indoor radio propagation.

- The channel bandwidth should be chosen as large as possible to suppress small-scale fading.

- For a proper RSS resolution, the distance between the mobile $\mathrm{BN}$ and the reference nodes shall be limited. This also limits the distance between the RNs and the overall coverage of the system.

\section{SENSOR BACKBONE COVERAGE}

To define the region which can be covered by the reference infrastructure, it is necessary to make some assumptions which are given by the ISO CAN specification [4] and the surrounding conditions of the application:

- The achievable location estimation accuracy is correlated to the distance between the reference nodes. Real life experiments have shown that useful system accuracies can be realized with node distances up to $3 \mathrm{~m}[3,5]$.

- The CAN cable length is three times the node distance. When the distance between the reference nodes is $3 \mathrm{~m}$, for a suitable mounting each CAN cable between the nodes has to be $9 \mathrm{~m}$ in length.

- A deterministic communication without large latencies is feasible with $50 \%$ bus load. Thus, the practical CAN data rate is two times the CAN gross data rate.

- For a more generalized applicability of the investigations, the maximum number connected nodes on a single CAN bus is limited to 32 . This criterion fits the demands of the electrical loads using typical low-speed and high-speed CAN transceivers.

With these predefined assumptions and the relationship between CAN cable length and CAN data rate (cf. Fig. 5) it is possible to define the maximum coverage of the one-dimensional localization system.

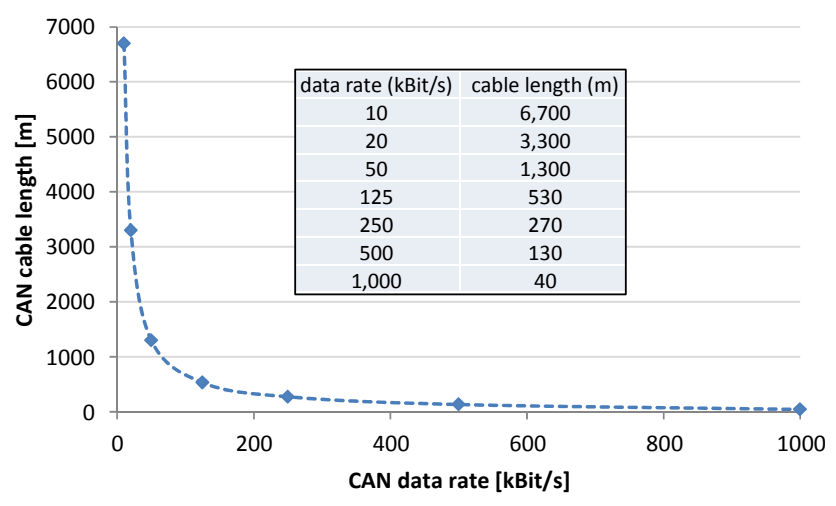

Fig. 5 - Maximum specified data rate and cable length for CAN bus lines [4].

The number of reference nodes (influencing the accuracy) and the number of simultaneously covered blind nodes affect the required data rate. The net data rate to collect the sensor information from $n$ blind nodes using $m$ reference nodes is given with

$$
D R_{n e t}=m\left[\frac{n x}{8}\right] f_{p o s} 64 \text { Bit , }
$$

where $x$ is the number of bytes for each sensor and $f_{\text {pos }}$ is the position update rate. For the CAN gross data rate, no generally valid value exists. The CAN header and inter frame gap have deterministic values. Since the number of stuff bits is dependent on the actual bit stream, the overall number of bits per CAN message is variable. A worst case scenario derived from the bit timing considerations in [25] leads to

$$
D R_{\text {gross }}=m\left[\frac{n x}{8}\right] f_{p o s}\left(\left[\frac{34+64}{4}\right]+47+64\right) \text { Bit }
$$


The practical data rate for a nearly deterministic communication rate depends on the specific application with allocation of more or less high prioritized message identifiers. The maximum tolerated bus load for the given application is $50 \%$ Thus, using (6) the practical data rate is two times the gross data rate.

$$
D R_{\text {det }} \geq \frac{D R_{\text {gross }}}{0.5}
$$

\subsection{CENTRALIZED LOCATION ESTIMATION}

The infrastructure components for the centralized location estimation are shown in Fig. 6. Up to 32 reference nodes (RNs) receive radio packets from up to 20 blind nodes (BNs) twice a second. The according RSS values are stored with one Byte and are transmitted to the data concentrator PC using a single CAN bus. In Fig. 6, an example distribution of the RSS values from all RNs is shown for a single BN located at RN 14.

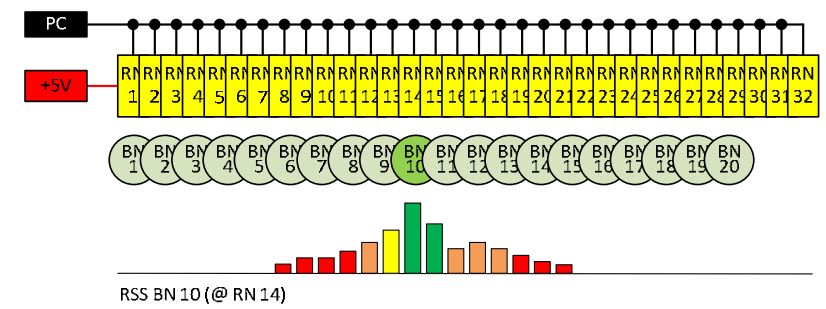

Fig. 6 - Infrastructure components for centralized location estimation of 20 blind nodes (BNs) using RSS values from 32 reference nodes (RNs).

A net data rate of $D R_{\text {net }}=12.3 \mathrm{kBit} / \mathrm{s}$ has to be realized by the CAN backbone using (4). The gross data rate with additional CAN header, bit stuffing and inter frame gap is $D R_{\text {gross }}=26.2 \mathrm{kBit} / \mathrm{s}$. As stated above, for an error-free communication without the suppression of less prioritized CAN packets, the data rate should be twice as large as the theoretical gross bus data rate. With $m=32$ RNs and $n=20$ mobile BNs, a practical CAN data rate of $D R_{\text {det }}=52.4 \mathrm{kBit} / \mathrm{s}$ leads to a specified CAN data rate of $D R_{C A N}=125 \mathrm{kBit} / \mathrm{s}$.

Thus, the theoretical maximum CAN cable length is $530 \mathrm{~m}$ with a corresponding maximum system coverage of approximately $177 \mathrm{~m}$. Furthermore, the maximum number of 32 connected nodes on a single CAN bus together with the given node distance of $3 \mathrm{~m}$ limit the overall system coverage to $96 \mathrm{~m}$.

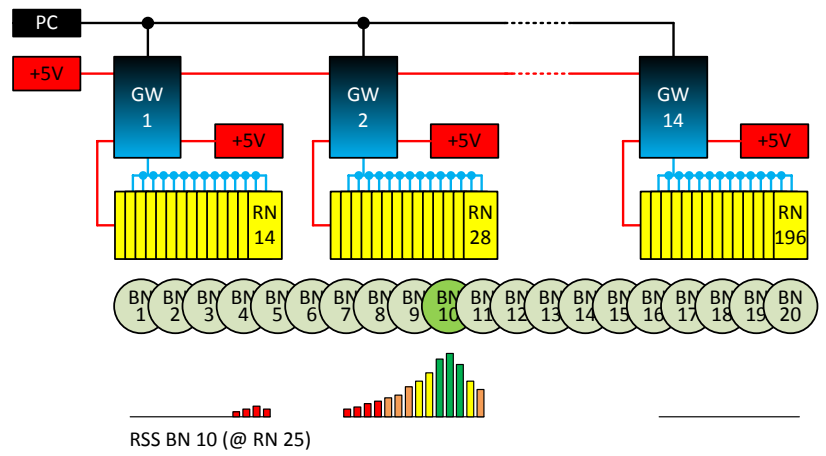

Fig. 7 - Hierarchical infrastructure for decentralized location estimation of 20 blind nodes (BNs) using 196 reference nodes (RNs) which are connected over 14 gateways (GWs).

\subsection{DISTRIBUTED LOCATION ESTIMATION}

To increase to coverage of the localization system, the distributed RSS localization uses additional infrastructure components to realize a hierarchical backbone bus. Up to 14 gateways with two CAN interfaces are used to connect up to 196 reference nodes to the data concentrator (cf. Fig. 7).

The embedded gateway architecture is shown in Fig. 8. To meet the requirements of ignition protection for the use in explosion-risk areas, the two CAN interfaces are electrically isolated. The CAN 1 transceiver has a separate $5 \mathrm{~V}$ power supply and is connected to the Cortex M4 system MCU via opto-couplers. Beside a large set of peripheral components, the MCU contains two dedicated CAN controllers.

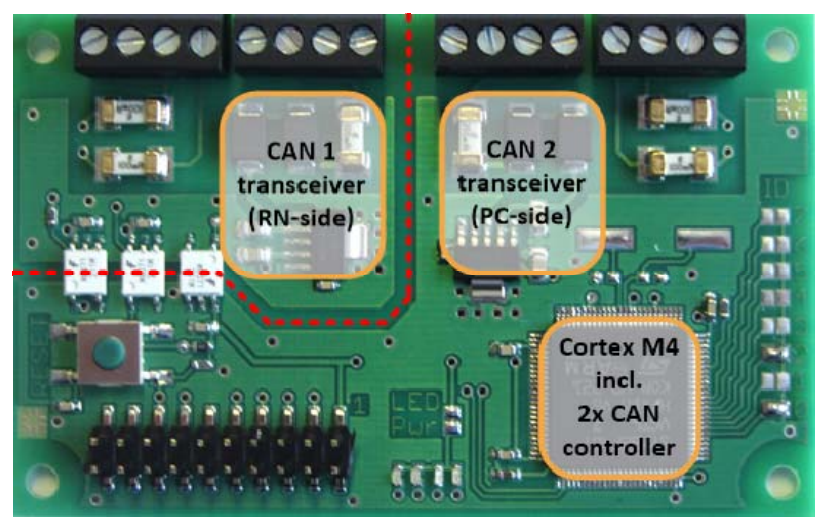

Fig. 8 - Embedded gateway architecture, top side of PCB showing CAN transceivers and Cortex M4 MCU.

For the centralized localization computation without data compression in the gateways, the PCbackbone CAN has to offer a minimum of $D R_{\text {net }}=75.3 \mathrm{kBit} / \mathrm{s}$. The theoretical gross bus data rate with additional CAN header and inter frame gaps has to be larger than $D R_{\text {gross }}=159.4 \mathrm{kBit} / \mathrm{s}$. 
With 196 RNs, organized into 14 gateway subnets and 20 mobile BNs, this results in a practical CAN data rate of $D R_{\text {det }}=318.8 \mathrm{kBit} / \mathrm{s}$. For the given scenario we have to set $D R_{C A N}=500 \mathrm{kBit} / \mathrm{s}$, which is defined in the CAN specification. Looking to Fig. 5, this data rate limits the maximum cable length to $130 \mathrm{~m}$. With this hierarchical bus concept, the maximum number of reference nodes can easily be scaled according to the application needs. Even though, the maximum cable length of $130 \mathrm{~m}$ for the top hierarchical bus-segment limits the system's coverage to less than $43 \mathrm{~m}$. For the given application example of localization in the underground mining, at least $300 \mathrm{~m}$ coverage along a line of reference nodes is required.

To obtain a larger coverage, the backbone cable length has to be increased and thus, the backbone data rate has to be decreased by the same order of magnitude. The required data compression with a distributed location computation on the gateways is described in the following. For each $\mathrm{BN}$, the gateways process the 14 byte RSS data (from all connected RNs) to compute a 1 byte subnet position information. Only the subnet position information are transmitted to the data concentrator PC. With this data compression by factor 14 , the required data rate of the top hierarchical bus-segment is reduced significantly.

For the given example, the data rate of all 15 CAN segments (14x RN-side, 1x PC-backbone) can be set to $D R_{\text {CAN }}=50 \mathrm{kBit} / \mathrm{s}$. Thus, the specified maximum cable length for each segment is $1,300 \mathrm{~m}$, resulting in an overall system coverage of $433 \mathrm{~m}$. Compared to the centralized localization computation the coverage of the localization system is increased by factor ten. The detailed location computation steps on the gateways and the PC will be described in detail in the following section.

\section{DISTRIBUTED WCL ALGORITHM}

In the previous chapter, we introduced a segmentation of the sensor data backbone with data compression in the gateways. The data compression requires a distributed computation of a subnet $\mathrm{BN}$ positions and an additional weighting factor, denoting the influence of each subnet on the final centralized computation.

At all gateways, the subnet $\mathrm{BN}$ position is calculated according to the SAWCL algorithm given in [26]. First, the distances between all receiving RNs and the $\mathrm{BN}$ are calculated using

$$
d_{i j}=10\left(\frac{R S S_{i j}-A}{10 n}\right) \text {, }
$$

where the reference signal strength $A$ and the path loss coefficient $n$ are taken from the corridor path loss experiment (cf. Fig. 4). The distances are used to compute a weight according to

$$
w_{i j}=\frac{1}{\left(d_{i j}\right)^{g}}
$$

A weighting factor of $g=3.0$ was found to be a good value for obstructed indoor environments [27].

The final calculation of the local position $d_{i}(x)$ of $\mathrm{BN} i$ is given by the weighted centroid of all RN positions $B_{j}(x)$ using

$$
p_{i}(x)=\frac{\sum_{j=1}^{m}\left(w_{i j} B_{j}(x)\right)}{\sum_{j=1}^{m} w_{i j}},\left\{p_{i} \in \mathbb{N} \mid 0<p_{i} \leq m\right\}
$$

This one-dimensional position is rounded to an integer value, representing the ID of the nearest RN. Thus, using $m=14$ RNs, the rough position fits in a 4 bit representation when it is transmitted to the data concentrator PC. Additionally, the gateways process the 1 byte RSS values from all $m$ connected RNs to compute a specific weighting factor $v_{i}$ with 4 bit representation. The weighting factor $v_{i}$ is calculated using the average RSS of all $m$ RNs according to

$$
v_{i}=\frac{S_{\min }-\frac{\sum_{j=1}^{m} R S S_{i j}}{m}}{S_{\min }-A} \cdot 15,\left\{v_{i} \in \mathbb{N} \mid 0<v_{i} \leq 15\right\} \text {, }
$$

where $A$ is the reference RSS in a distance of $1 \mathrm{~m}$ and $S_{\min }$ is the receiver's sensitivity level. The value of $v_{i}$ is normalized to a range of $0 . .15$ to fit into the 4 bit representation.

The gateways forward the compressed data $p_{i}(x)$ and $v_{i}$ to the data concentrator PC over the backbone CAN. On the PC the information from all gateways are processed to calculate the final position $P_{i}(x)$ of the mobile BN with ID $i$ using

$$
P_{i}(x)=\frac{\sum_{j=1}^{q}\left(v_{i}^{\prime} p_{i}(x)\right)}{\sum_{j=1}^{q} v_{i}^{\prime}}
$$

The impact of each segment position $p_{i}(x)$ can be tuned by modifying the weight $v_{i}$ according to 


$$
v_{i}^{\prime}=v_{i}^{k},
$$

where $k$ is an additonal weighting factor. Thus, it is possible to adapt the position estimation algorithm to different scenarios with varying infrastructure node setups. Beside the node distance, the ratio between the overall number of nodes and the nodes per subnet influence the optimum value of $k$.

\section{TRACKING SIMULATION}

For detailed investigations of different bus topologies, a simulation of the distance depending RSS is better suited than experiments, since the setup for hundreds of reference nodes would take a lot of time and space. Furthermore, real world experiments also limit the modification of process parameters (e.g. number of gateways), since the raw values are already processed on the gateways and only position information are available at the data concentrator PC. Thus, one set of measurement values cannot be analyzed with different gateway utilizations. For a parameterized computation using simulations of RSS distributions this is not an issue.

For the distance depending RSS we take the path loss model shown in Fig. 4. The average path loss is given with the log-distance model according to (1). For the varying part of the model we assume Rician fading using (2) and a Rician K-factor of $17.7 d B$. As shown in the application's environment in Fig. 1, there exist a dominating LOS component of the superimposed RSS and only a few NLOS conditions might occur (e.g. shadowing due to human body). Thus, Rician fading is more suitable than Raleigh fading to model the multipath fading channel [18].

The infrastructure for the tracking simulation contains a line of 196 reference nodes $1 \mathrm{~m}$ beside the track and with $1 \mathrm{~m}$ node-to-node distance. A mobile blind node moves with a speed of $0.5 \mathrm{~m} / \mathrm{s}$ along the track and periodically sends out $2.4 \mathrm{GHz} \mathrm{RF}$ packets every $2 \mathrm{~Hz}$. The reference RSS $A$ in a distance of $1 \mathrm{~m}$ is set to $-67 \mathrm{dBm}$ and the receiver's sensitivity $S_{\min }$ is $-110 \mathrm{dBm}$.

In Fig. 9, the RSS values of all 196 reference nodes are plotted for a time duration of 392 s (784 iterations) and a blind node's linear motion from RN 1 to RN 196 (195 m overall track length). The color of the scatter plot indicates the RSS, where red corresponds to a high value near the reference path loss and dark blue to a relatively low RSS. Note that the z-axis starts at $S_{\text {min }}=-110 \mathrm{dBm}$ and all values below this threshold are clipped off and neglected for the simulation model.

To figure out the influence of the distributed location estimation, two different utilizations are compared in the following. A centralized WCL computation (cWCL), corresponding to $\mathrm{m}=1$ gateway and a distributed computation (dWCL) with $\mathrm{m}=14$ gateways.

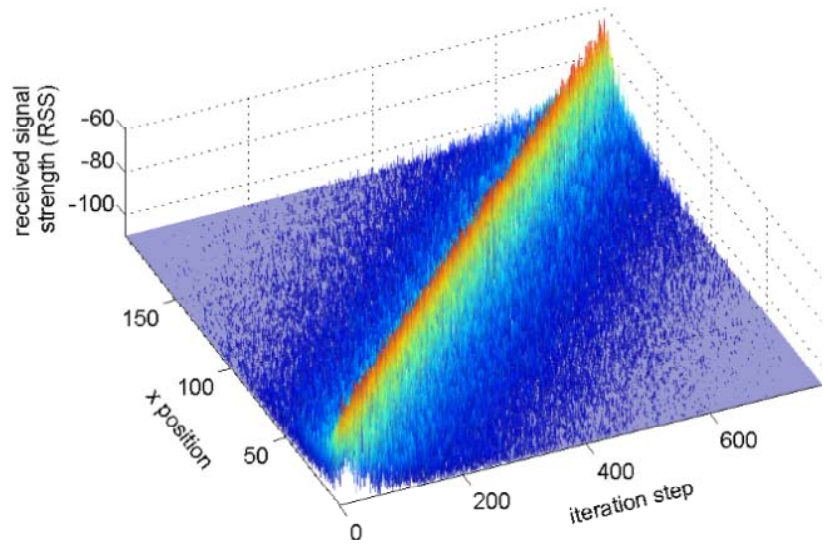

Fig. 9 - Received signal strength distribution for onedimensional tracking simulation of a mobile blind node (BN) along 196 reference nodes (RNs).

The location estimation error (LEE) is given by the Euclidean distance between the estimated position and the true position, which is the absolute difference of $\mathrm{x}$-positions in the one-dimensional case as given with

$$
\operatorname{LEE}(x)=\|\hat{x}-x\|_{2}=|\hat{x}-x|
$$

In Fig. 10, the accuracy of both configurations is compared for the partial track between RN 37 and RN 87, covering three complete subnets of 14 reference nodes in the distributed localization scenario.

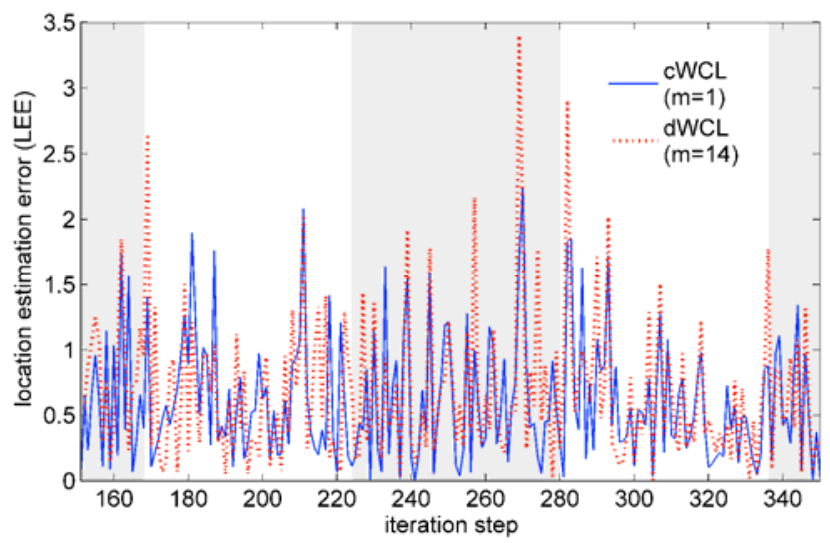

Fig. 10 - Location estimation error (LEE) for onedimensional tracking simulation of a mobile blind node (BN). Comparison of centralized computation (cWCL) and distributed computation (dWCL) with $\mathbf{m = 1 4}$ gateways (extract showing 200 iteration steps for BN movement from RN 37 to $R N$ 87, passing four gateway segment borders). 
For most of the position estimations in the middle of a subnet there are only small differences between the two configurations. At the segment borders, the distributed computation has higher errors than the centralized computation.

A detailed comparison of both configurations is given with the error cumulative distribution functions in Fig. 11 and detailed error statistics in Table 2.

Table 2. Comparison of 3-sigma location estimation error (in $\mathbf{m}$ ) for centralized and distributed weighted centroid localization.

\begin{tabular}{|c|c|c|c|c|}
\hline error & $\begin{array}{c}\text { cWCL } \\
\mathrm{m}=1\end{array}$ & $\begin{array}{c}\mathrm{dWCL} \\
\mathrm{m}=1\end{array}$ & $\begin{array}{c}\mathrm{dWCL} \\
\mathrm{m}=4\end{array}$ & $\begin{array}{c}\text { dWCL } \\
\mathbf{m = 1 4}\end{array}$ \\
\hline median & 0.48 & 0.50 & 0.50 & $\mathbf{0 . 5 4}$ \\
\hline sigma & 0.22 & 0.26 & 0.39 & $\mathbf{0 . 3 3}$ \\
\hline 3-sigma & 2.78 & 3.00 & 3.70 & $\mathbf{3 . 1 4}$ \\
\hline maximum & 3.56 & 4.00 & 5.54 & $\mathbf{4 . 1 0}$ \\
\hline
\end{tabular}

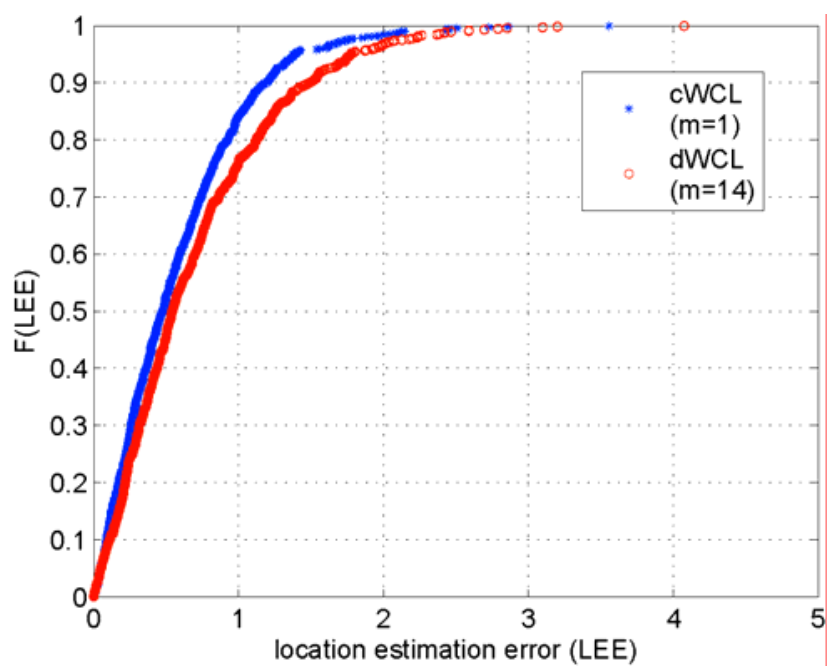

Fig. 11 -Cumulative location estimation error for $196 \mathrm{~m}$ linear track (empirical value), comparing centralized computation (cWCL) and distributed computation (dWCL) with $\mathbf{m}=14$ gateways.

The influence of the number of gateways is analyzed in Fig. 12, where the 3-sigma error and the achievable coverage are shown for up to 28 gateways. The optimum accuracy for dWCL is reached with $\mathrm{m}=14$ gateways. With the considerations in section 4, a CAN data rate of $50 \mathrm{kBit} / \mathrm{s}$ is sufficient to transmit all necessary sensor information. Looking at Fig. 5, a localization coverage along a line of $433 \mathrm{~m}$ can be realized with this setup. With a centralized WCL computation a specified CAN data rate of $500 \mathrm{kBit} / \mathrm{s}$ would be required which limits the system coverage to $43 \mathrm{~m}$. This corresponds to a coverage enhancement by factor 10, when a distributed computation is used instead of a centralized one. At the same time, the maximum (3-sigma) error for the distributed computation with $\mathrm{m}=14$ gateways compared to the conventional centralized one increases by $15.17 \%$ (12.95\%).

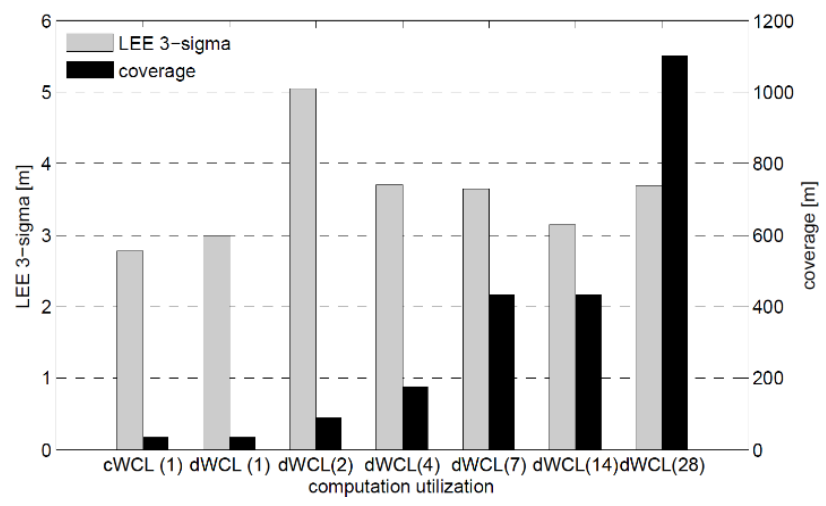

Fig. 12 -Comparison of the centralized (cWCL) and distributed (dWCL) weighted centroid location estimation, using 3-sigma location estimation error and one-dimensional localization coverage.

The influence of the weighting factor $k$ on the location estimation error is analyzed in Fig. 13, where the cumulative error is shown for $1 \leq k \leq 50$. Additionally, the 3-sigma location estimation error is compared in Table 3 for selected values.

Table 3. 3-sigma location estimation error (in $\mathrm{m}$ ) for different weighting factor $\mathbf{k}$ using distributed weighted centroid localization with $\mathbf{m}=14$ gateways.

\begin{tabular}{|c|c|c|c|c|c|c|}
\hline Error & $\mathrm{k}=2$ & $\mathrm{k}=4$ & $\mathbf{k}=\mathbf{6}$ & $\mathrm{k}=8$ & $\mathrm{k}=10$ & $\mathrm{k}=12$ \\
\hline 3-sigma & 7.25 & 3.16 & $\mathbf{3 . 1 4}$ & 3.31 & 3.69 & 3.95 \\
\hline
\end{tabular}

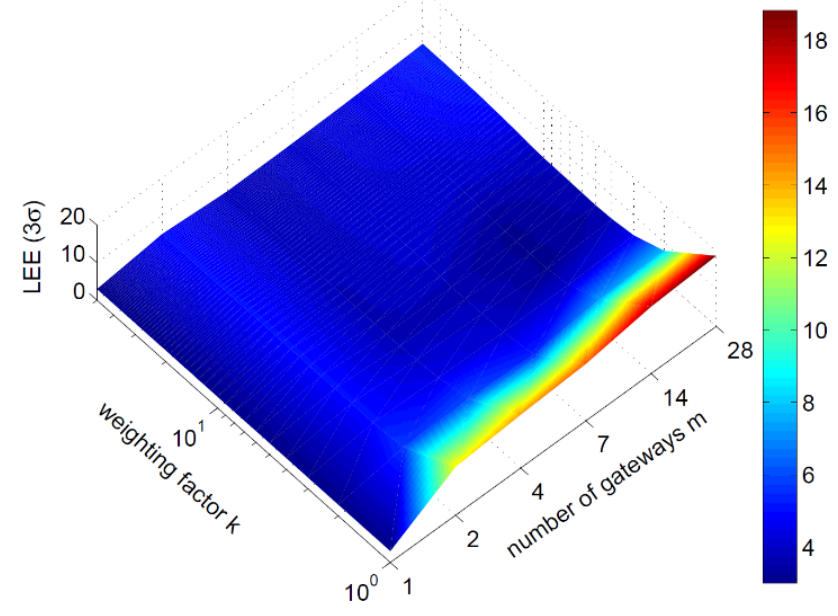

Fig. 13 - Combined influence of the number of gateways $m$ and the weighting factor $k$ on the 3-sigma location estimation error.

In the case a BN is located near the middle of a gateway segment, the position information from neighboring segments negatively influence the location estimation. This effect can be reduced by selecting an appropriate weighting factor $k$. For $k \leq 2$ there exist large position errors, showing the 
negative influence from neighboring gateway segments. The lowest error is reached with $k=6$, where the influence from neighboring segments is shifted more to the segment borders. Larger values of $k$ slightly decrease the estimation accuracy, since small systematic errors are introduced at the gateway segment borders.

\section{CONCLUSION AND FUTURE WORK}

The distributed WCL localization based on RSS readings is proposed to increase the system's coverage, limited by bus load constraints for collecting all sensor information. The results from a one-dimensional tracking simulation show, that it is possible to split up the location estimation into distributed parts of computation. Compared to the cWCL estimation, the increase in location estimation error for dWCL is relatively low and acceptable for most applications.

The main contribution of the decentralized location computation is a considerably increase of the tracking system's coverage up to the kilometers range. The system also can easily be scaled to the application's needs. An example scenario for personnel tracking in the underground longwall mining with 196 reference nodes shows a good localization performance for a utilization with 14 gateway segments. The corresponding system coverage of more than $400 \mathrm{~m}$ is sufficient for real world underground longwall mining setups.

Further system developments are focusing on enhancements of the dWCL algorithm, especially to encounter some systematic computation errors at the edge of the regarding track. The second issue for future work is a real life tracking measurement in the underground to compare the propagation effects with the corridor experiment and the corresponding assumptions for the simulated RSS distribution.

\section{REFERENCES}

[1] Z. Sun and I. F. Akyildiz, Channel modeling and analysis for wireless networks in underground mines and road tunnels, IEEE Transactions on Communication, (58) 6 (2010), pp. 1758-1768.

[2] J. Figueiras and S. Frattasi, Mobile Positioning and Tracking - From Conventional to Cooperative Techniques, 2010.

[3] A. Fink and H. Beikirch, Adding link quantity information to redundant RF signal strength estimates for improved indoor positioning, $3^{r d}$ IEEE International Conference on Indoor Positioning and Indoor Navigation (IPIN), 2012, pp. 1-6.
[4] ISO 11898-1, Road Vehicles - Controller Area Network - Part 1: Data Link Layer and Physical Signaling, ISO Std., 2003.

[5] A. Fink and H. Beikirch, Combining of redundant signal strength readings for an improved RF localization in multipath indoor environments, $15^{\text {th }}$ International Conference on Information Fusion, 2012, pp. 1-7.

[6] A. Fink and H. Beikirch, Radio-based human tracking for large indoor environments using distributed centroid location estimation, $7^{\text {th }}$ IEEE International Conference on Intelligent Data Acquisition and Advanced Computing Systems (IDAACS), 2013, pp. 442-449.

[7] R. Mautz, Indoor Positioning Technologies, Habilitation Thesis, 2012.

[8] T. K. Kohoutek, R. Mautz, and A. Donaubauer, Real-time indoor positioning using range imaging sensors, Proceedingsd of SPIE Photonics, (7724) (2010).

[9] M. Allen, S. Baydere, E. Gaura, and G. Kucuk, Evaluation of localization algorithms, Localization Algorithms and Strategies for Wireless Sensor Networks: Monitoring and Surveillance Techniques for Target Tracking, 2009, pp. 348-379.

[10] P. Barsocchi, S. Chessa, and F. Furfari, Evaluating AAL solutions through competitive benchmarking: the localization competition. IEEE Pervasive Computing, (99) (2013).

[11] A. Fink, H. Beikirch, and M. Voss, Improved indoor localization with diversity and filtering based on received signal strength measurements, International Journal of Computing, (9) 1 (2010).

[12] T. S. Rappaport, Wireless Communications Principles and Practice, Prentice Hall PTR, 2002.

[13] H. Benn and H. Thomas, GSM in the indoor business environment, in GSM Evolution Towards 3rd Generation Systems, Z. Zyonar, P. Jung, and K. Kammerlander, Eds. Kluwer Academic Publishers, 2002, pp. 211-234.

[14] D. Cheung and C. Prettie, A path loss comparison between the $5 \mathrm{GHz}$ UNII band (802.11a) and the $2.4 \mathrm{GHz}$ ISM band (802.11b). Intel Labs, Technical Report, 2002.

[15] T. S. Rappaport and C. D. McGillem, UHF fading in factories, IEEE Journal of Selected Areas Communications, (7) 1 (1989), pp. 40-48.

[16] Texas Instruments, CC2500: low-cost lowpower $2.4 \mathrm{GHz} R F$ transceiver. rev. C, 2008.

[17] C. Beder, A. McGibney, and M. Klepal, Predicting the expected accuracy for fingerprinting based WiFi localisation systems, $2^{\text {nd }}$ IEEE International Conference on Indoor 
Positioning and Indoor Navigation (IPIN), 2011, pp. 1-6.

[18] A. Abdi, C. Tepedelenlioglu, M. Kaveh, and G. Giannakis, On the estimation of the parameter for the rice fading distribution, IEEE Communication Letteers, (5) 3 (2001), pp. 92-94.

[19] A. T. Parameswaran, M. I. Husain, and S. Upadhyaya, Is RSSI a reliable parameter in sensor localization algorithms, an experimental study, IEEE International Symposium on Reliable Distributed Systems, 2009.

[20] E. Elnahrawy, X. Li, and R. P. Martin, The limits of localization using signal strength: A comparative study, IEEE Sensor and Ad Hoc Communications and Networks (SECON), 2004, pp. 406-414.

[21] K. Whitehouse, C. Karlof, and D. Culler, A practical evaluation of radio signal strength for ranging-based localization, SIGMOBILE Mobile Computing and Communications Review, (11) (January 2007), pp. 41-52.

[22] G. Chandrasekaran, M. A. Ergin, J. Yang, S. Liu, Y. Chen, M. Gruteser, and R. P. Martin, Empirical evaluation of the limits on localization using signal strength, IEEE Sensor and Ad Hoc Communications and Networks (SECON), 2009.

[23] Y. P. Zhang, G. X. Zheng, and J. H. Sheng, Radio propagation at $900 \mathrm{MHz}$ in underground coal mines, IEEE Transactions on Antennas Propagation, (49) 5 (2001), pp. 757-762.

[24] M. Boutin, A. Benzakour, C. Despins, and S. Affes, Characterization and modeling of a wireless channel at 2.4 and $5.8 \mathrm{GHz}$ in underground tunnels, 3rd International
Symposium on Wireless Communication Systems (ISWCS), 2006, pp. 517-521.

[25] K. Tindell and A. Burns, Guaranteed message latencies for distributed safety-critical hard real-time control networks. Technical Report, 1994.

[26] A. Fink and H. Beikirch, RSSI-based indoor localization using antenna diversity and plausibility filter, $6^{\text {th }}$ Workshop on Positioning Navigation and Communication, 2009, pp. 159-165.

[27] A. Fink and H. Beikirch, Analysis of RSSbased location estimation techniques in fading environments, $2^{\text {nd }}$ IEEE International Conference on Indoor Positioning and Indoor Navigation (IPIN), 2011, pp. 1-6.

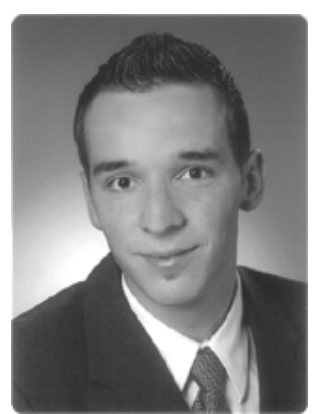

Andreas Fink, received his M.Sc. degree in Information Technology from Rostock University in 2008. Currently, he works as a research scientist at the Department of Computer Science and Electrical Engineering at Rostock University. His primary research interests focus on wireless communication sys-tems, radio-based indoor positioning and hybrid localization with inertial sensors.

Helmut Beikirch, to hold the professorship "Electronic Devices and Circuit Design" at the Department of Computer Science and Electrical Engineering at Rostock University since 1998. He is the head of the research group in electronic circuit design and communication systems in industrial automation. 